\title{
Scattering from a Slit Composite Cylindrical Shell
}

\author{
Cheng-Nan Chiu, Hsiao-Chang Chu, and Chun Hsiung Chen \\ Department of Electrical Engineering \\ National Taiwan University \\ Taipei 10617, Taiwan, R.O.C.
}

\begin{abstract}
A new theory based on differential equation approach is proposed for discussing the electromagnetic fields in a multi-layered and anisotropic cylindrical shell with an infinitely long slot which is illuminated normally by a plane wave. By this theory, numerical results of monostatic and bistatic scattering are presented for slit graphite/epoxy fiber-reinforced composite cylindrical shell. In particular, the shells with different slot angles as well as the plane waves with both TE and TM polarizations which are incident from different directions are investigated in detail.
\end{abstract}

\section{Introduction}

Electromagnetic scattering from a slit conducting cylindrical structure has been extensively studied, because the apertures on aircrafts may affect the scattering characteristics.

Advanced composite materials have been widely used in modern aircraft systems due to their superior mechanical properties. A thorough investigation on shielding and reflection properties of planar graphite/epoxy $(\mathrm{G} / \mathrm{E})$ and boron/epoxy $(\mathrm{B} / \mathrm{E})$ composite was given by $L i n$ et al. [1]. However, the curvature, creeping wave, and radial resonance effects which can occur in the cylindrical structures were not discussed in these studies. Therefore, further investigation on shielding and scattering phenomena for $G / E$ and $B / E$ cylindrical shells was conducted by Chiu and Chen [2],[3].

The purpose of this study is to present a scattering analysis of $\mathrm{G} / \mathrm{E}$ composite cylindrical shell structure with an infinite slot (Fig. 1). To this end, a new theory for characterizing the electromagnetic fields in a slit, multi-layered, lossy, and anisotropic cylindrical shell is proposed. Particularly, the monostatic and bistatic echo widths of the slit composite cylindrical shells are presented and discussed.

\section{Formulation}

Consider a two-dimensional problem (Fig. 1) in which a plane wave is normally incident upon an N-layer anisotropic cylindrical shell. Suppose that this shell has an infinite slot af angle $2 \phi_{m}$. The composite shell structure may be discussed by the complex permeability and permittivity tensors [2],[3]. Then the governing equations may be established, using a differential equation approach and a finite difference approximation.

In this study, the echo widths for the TM to $\mathrm{z}$ and TE to $\mathrm{z}$ cases are defined as

$$
e_{e} W_{e}=\lim _{\rho \rightarrow \infty} 2 \pi \rho\left|E_{z}^{s} / E_{z}^{i}\right|^{2}
$$




$$
{ }_{m} W_{m} \lim _{\rho \rightarrow \infty} 2 \pi \rho\left|H_{z}^{s} / H_{z}^{i}\right|^{2}
$$

where the symbols "i" and " $\mathrm{s}$ " are associated with the incident and scattered fields, and the subscripts " $\mathrm{e}$ " and " $\mathrm{m}$ " are with the electric and magnetic fields, respectively.

\section{Results}

Graphite/epoxy (G/E) fiber-reinforced laminated composites are the main concern of this study. This material is non-magnetic and whose constitutive parameters are: $\sigma_{\|}=4 \times 10^{4} \mathrm{~S} / \mathrm{m}, \sigma_{\perp}=200 \mathrm{~S} / \mathrm{m}, \epsilon_{r}=5.0$. Here, the symbols " $\|$ " and " $\perp$ " denote the directions parallel and perpendicular to the fiber, respectively. We here consider the composite cylindrical shell structure with layer thickness $d \equiv d_{1}=d_{2}=\cdots d_{N}=0.127$ $\mathrm{mm}$, and interior radius $a \equiv \rho_{N}=2 \mathrm{~m}$. In all computations, the plane wave is assumed to be normally incident with an angle $\phi_{0}$ with respect to the $\mathrm{x}$-axis. The fiber orientation pattern is limited to the $[0 / 45 / 90 /-45]$ one.

Shown in Fig. 2 is the relationship between monostatic echo width and $k a$ for [0/45/90/-45] G/E cylindrical shells with the half slot angle $\phi_{m}$ as parameters. Here, the slot is directly illuminated $\left(\phi_{0}=0\right)$. A comparison of echo widths for the slit G/E cylindrical shell with half slot angle $\phi_{m}=90$ illuminated from different directions is shown in Fig. 3. The scattering characteristics of the slit shell illuminated from $\phi_{0}=180$ is very similar to that of the corresponding closed one $\left(\phi_{m}=\phi_{0}=0\right)$. The bistatic scattering is shown in Fig. 4. The reciprocal properties are preserved for incidence from $\phi_{0}=0$ and 180 .

\section{Conclusion}

The scattering is influenced by the shell size, the material properties, the creeping wave on the exterior surface, the resonance phenomenon in the hollow region, the slot angle, and the polarization as well as the illuminated angle of the incident wave. The characteristics of scattering from a slit G/E cylindrical shell are similar to that of a slit PEC cylindrical shell except that the "low-frequency scattering mode" is not excited. The interior or exterior loaded cylindrical shells are suggested for reducing the echo widths for G/E slit cylindrical shells.

Acknowledgment This study was supported by the National Science Council of Taiwan, Republic of China under Grant NSC 85-2213-E-002-001.

\section{References}

[1 ] M.-S. Lin and C. H. Chen, "Plane-wave shielding characteristics of anisotropic laminated composites," IEEE Trans. Electromagn. Compat, vol. 35, no. 1, pp. 21-27, Feb. 1993 .

[2 ] C.-N. Chiu and C. H. Chen, "Plane-wave shielding properties of anisotropic laminated composite cylindrical shells," IEEE Trans. Electromagn. Compat., vol. 37, no. 1, pp. 109-113, Feb. 1995.

[3 ] -, "Scattering from an advanced composite cylindrical shell," IEEE Trans. Electromagn. Compat. vol. 38, no.1, pp. xxx-xxx, Feb. 1996. 


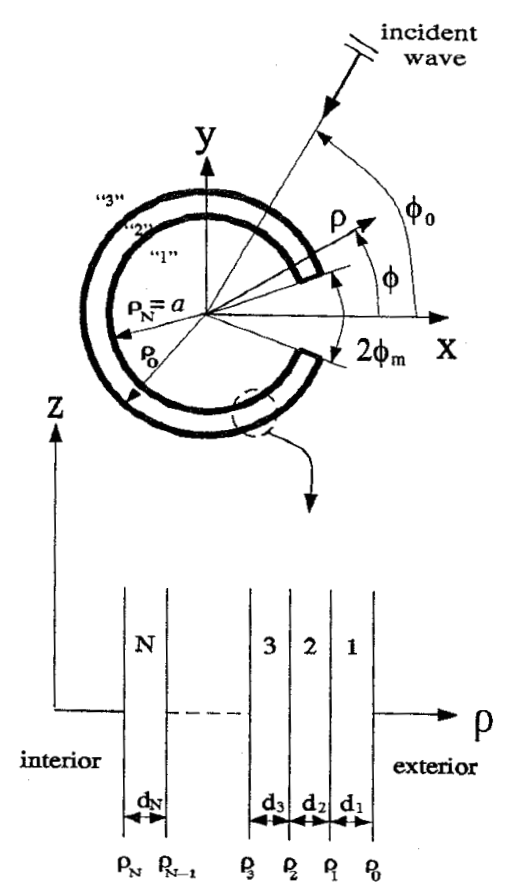

Fig. 1 Geometry of slit, multi-layered, and anisotropic cylindrical shell.
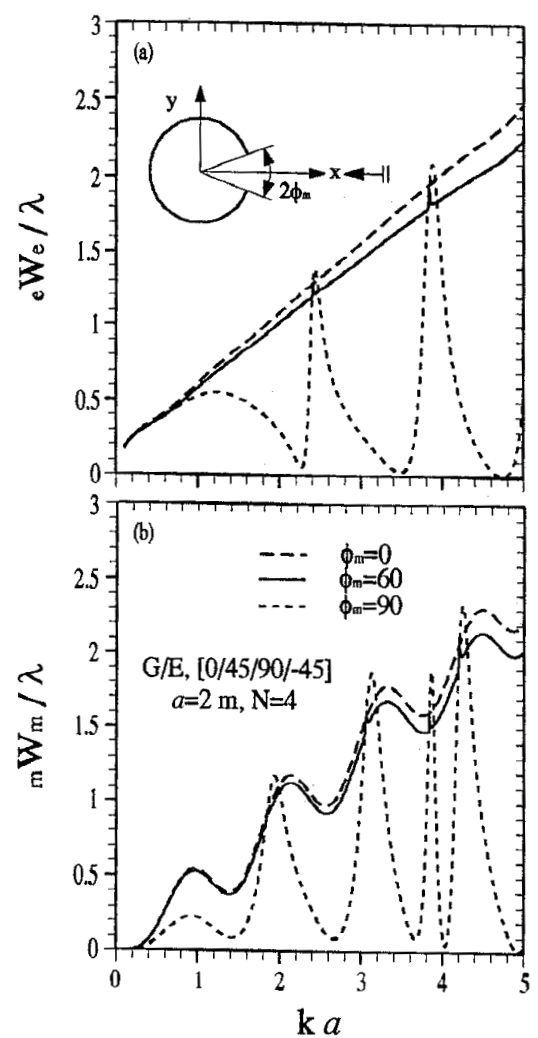

Fig. 2 Monostatic echo widths of slit [0/45/90/-45] G/E cylindrical shells with $\phi_{\mathrm{I}}$ (half slot angle) as parameters. $\left(a=2 \mathrm{~m}, \mathrm{~d}=0.127 \mathrm{~mm}, \mathrm{~N}=4 ; \phi_{0}=0\right.$.)

(a) TM wave, (b) TE wave. 


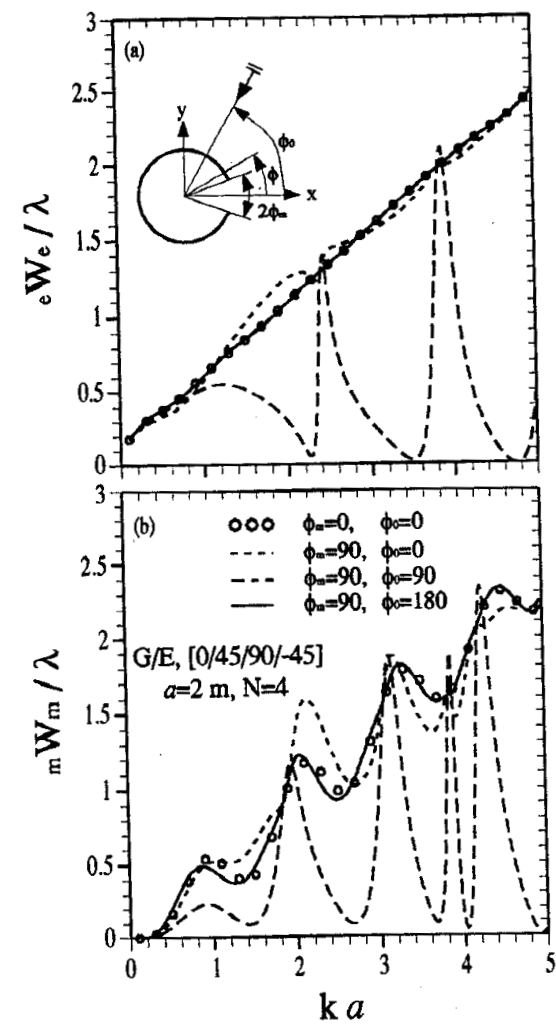

Pig. 3 Monostatic echo widths of slit [0/45/90/-45] G/E cylindrical shells with $\phi_{0}$ (incident angle) as parameters.

$\left(a=2 \mathrm{~m}, \mathrm{~d}=0.127 \mathrm{~mm}, \mathrm{~N}=4, \phi_{\mathrm{m}}=90\right.$.)

(a) TM wave, (b) TE wave.
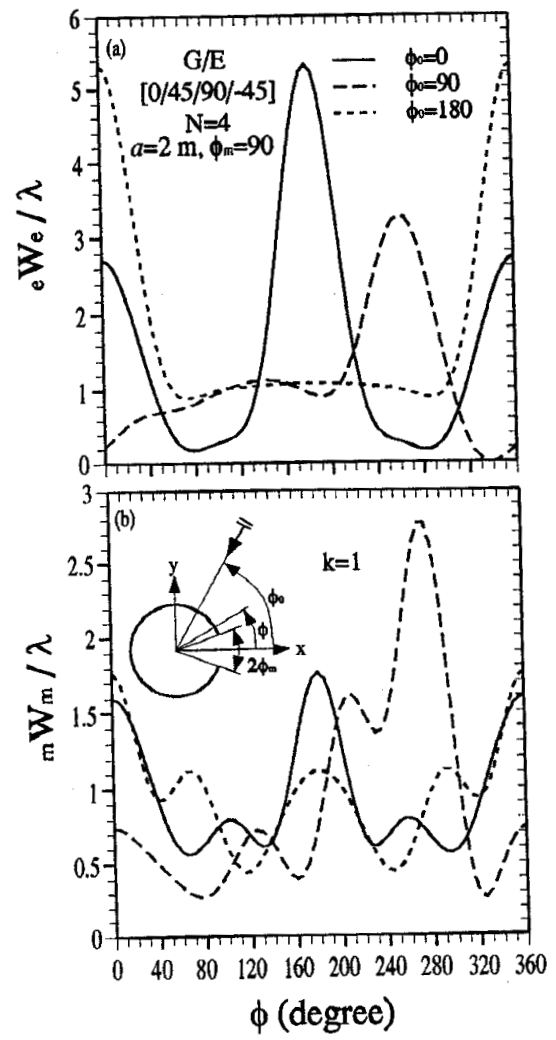

Fig. 4 Bistatic echo widths of slit $[0 / 45 / 90 /-45]$ $\mathrm{G} / \mathrm{E}$ cylindrical shells with $\phi_{0}$ as parameters. ( $a=2 \mathrm{~m}, \mathrm{~d}=0.127 \mathrm{~mm}, \mathrm{~N}=4, \phi_{n}=90 ; \mathrm{k}=1$.)

(a) TM wave, (b) TE wave. 